\title{
Critérios auditáveis para implementação de melhores práticas na adesão ao checklist cirúrgico
}

Auditable criteria for implementing best practices in adhering to the surgical safety checklist Criterios de auditoría para la implementación de mejores prácticas en la adhesión a la checklist quirúrgico

Danielle Bezerra Cabral ${ }^{1}$ (i) https://orcid.org/0000-0002-1760-4113

Mágda Letícia Pedroso Pereira ${ }^{1}$ id https://orcid.org/0000-0001-8382-9540

Michele Suzana Fernandes ${ }^{2}$ iD https://orcid.org/0000-0003-4095-7846

Suellen Fincatto ${ }^{1}$ iD https://orcid.org/0000-0001-7937-6876

Adriana Gracietti Kuczmainski ${ }^{1}$ io https://orcid.org/0000-0003-4476-438X

Arnildo Korb1 io https://orcid.org/0000-0001-7333-0754

Cabral DB, Pereira ML, Fernandes MS, citar:

S, Kuczmainski AG, Korb A. Critérios auditáveis

para implementação de melhores práticas

na adesão ao checklist cirúrgico. Acta Paul Enferm. 2021;34:eAPE00515.

DOI

http://dx.doi.org/10.37689/actaape/2021A000515

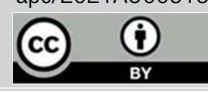

Descritores

Lista de checagem; Centros cirúrgicos; Seguranç̧a do paciente; Enfermagem perioperatória; Qualidade

da assistência à saúde

Keywords

Checklist; Surgicenters; Patient safety; Perioperative nursing; Quality of health care

Descriptores

Lista de verificación; Centros quirúrgicos; Seguridad del paciente; Enfermería perioperatoria;

Calidad de la atención de salud

Submetido

18 de Março de 2020

Aceito

15 de Setembro de 2020

\section{Autor correspondente}

Danielle Bezerra Cabral Email: danielle.cabral@udesc.br

\section{Resumo}

Objetivo: Avaliar a adesão ao uso da Lista de Verificação de Segurança Cirúrgica de um hospital, conforme os critérios auditáveis do Instituto Joanna Briggs.

Métodos: Estudo observacional descritivo que utilizou as ferramentas Practical Application of Clinical Evidence Systeme Getting Reasearch into Practice nas três fases previstas da metodologia: auditoria de base, educativa e auditoria de seguimento. As auditorias aconteceram no centro cirúrgico de um hospital do oeste de Santa Catarina. Na auditoria de base, foram elegíveis cem cirurgias, sendo as mesmas cem incluídas na auditoria de seguimento, com observação direta da equipe cirúrgica no preenchimento completo da Lista de Verificação de Segurança Cirúrgica.

Resultados: Na auditoria de base, os critérios 2 e 3 tiveram alta conformidade com a melhor prática (81\% a 93\%), e os critérios 1 e 4 (45\%, 49\%) apresentaram baixa conformidade. A partir das barreiras encontradas na auditoria de base, foram realizadas atividades educativas junto à equipe cirúrgica. Na auditoria de seguimento, houve aumento nos percentuais da conformidade em todos os critérios, e os critérios 2 e 3 obtiveram 100\% e 99\%, respectivamente, contudo os critérios 1 e 4 continuaram com percentuais abaixo do esperado (65\% e $54 \%$, respectivamente).

Conclusão: A não obtenção de 100\% de conformidade nos critérios auditados é ocasionada por um cuidado assistido não baseado na prática por evidências. Contudo, espera-se uma educação permanente e continuada para realizar as boas práticas na instituição do estudo.

\section{Abstract}

Objective: To assess adherence to the use of the hospital's Surgical Safety Checklist according to the auditable criteria of the Joanna Briggs Institute.

Methods: An observational descriptive study was conducted, using the Practical Application of Clinical Evidence System and Getting Research into Practice tools in the three phases of the study methodology: basic, educational and follow-up audits. The audits took place in the surgical center of a hospital in western Santa Catarina, Brazil. A total of 100 surgeries were eligible in the baseline audit, with the same 100 being included in the follow-up audit, and direct observation being performed by the surgical team in completing the Surgical Safety Checklist.

Results: Criteria 2 and 3 were highly compliant with best practices (81\% to 93\%) in the baseline audit, while Criteria 1 and 4 (45\%, 49\%) had low compliance. Thus, educational activities were conducted with the surgical team based on the barriers found in the basic audit. There was an increase in the compliance percentages for all criteria in the follow-up audit, and Criteria 2 and 3 obtained 100\% and 99\%, respectively; however, Criteria 1 and 4 continued with percentages below the expected ( $65 \%$ and $54 \%$, respectively). 
Conclusion: Failure to achieve $100 \%$ compliance with the audited criteria is caused by the provided care not being based on evidence-based practice. However, permanent and continuous education is expected to result in good practices at the study institution.

\section{Resumen}

Objetivo: Evaluar la adhesión al uso de la Lista de Verificación de Seguridad Quirúrgica de un hospital, según los criterios de auditoría del Instituto Joanna Briggs.

Métodos: Estudio observacional descriptivo que utilizó las herramientas Practical Application of Clinical Evidence System y Getting Reasearch into Practice en las tres fases previstas de la metodología: auditoría basal, educativa y auditoría de seguimiento. Las auditorías se realizaron en el quirófano de un hospital del oeste del estado de Santa Catarina. En la auditoría basal, se seleccionaron 100 cirugías y las mismas 100 se incluyeron en la auditoría de seguimiento, con observación directa del equipo quirúrgico para el llenado completo de la Lista de Verificación de Seguridad Quirúrgica.

Resultados: En la auditoría basal, los criterios 2 y 3 tuvieron alta conformidad con la mejor práctica (81 \% a 93 \%), y los criterios 1 y 4 (45 \%, 49 \%) presentaron baja conformidad. A partir de los obstáculos encontrados en la auditoría basal, se realizaron actividades educativas con el equipo quirúrgico. En la auditoría de seguimiento, hubo un aumento en los porcentajes de conformidad en todos los criterios. El criterio 2 obtuvo $100 \%$ y el 3, 99 \%; no obstante, los criterios 1 y 4 continuaron con porcentajes inferiores a lo esperado (65\% y $54 \%$, respectivamente).

Conclusión: La no obtención del 100 \% de conformidad en los criterios auditados se debe a un cuidado asistido no basado en la práctica por evidencias. Sin embargo, se espera una educación permanente y continua para realizar las buenas prácticas en la institución del estudio.

\section{Introdução}

O cuidado anestésico-cirúrgico é permeado por contextos dinâmicos, os quais são desenvolvidos por práticas de alto nível de complexidade, atendimento de variadas situaçóes e intervençóes invasivas, plenas de variação, mediadas pela interação de equipes interdisciplinares, exercidas em condiçóes ambientais dominadas pela agilidade, precisão e pressão. Essas características, muitas vezes, conduzem a condiçóes/situaçóes inseguras, definidas pela Organização Mundial da Saúde (OMS) como evento ou circunstância evitável, decorrente do cuidado, não associado à doença de base. ${ }^{(1,2)}$

Não obstante os inquestionáveis benefícios da abordagem no processo saúde-doença, a atenção cirúrgica está associada, consideravelmente, a importantes riscos de complicaçóes e morte. É nessa perspectiva, com vistas à redução na ocorrência de eventos adversos em pacientes submetidos a procedimentos cirúrgicos, que a OMS, em 2009, estabeleceu o segundo desafio global de segurança do paciente, a campanha Safe Surgery Saves Lives, divulgando diretrizes para a implantação de um protocolo universal de segurança do paciente cirúrgico, incluindo a definição da Lista de Verificação de Segurança Cirúrgica ou checklist. ${ }^{(1,3)}$

A Lista de Verificação de Segurança Cirúrgica é uma ferramenta estruturada em uma lista formal, utilizada para identificar, comparar e verificar um grupo de procedimentos nas três fases: antes do início da anestesia (sign in), antes da incisáo na pele (time out) e antes da saída do paciente da sala cirúrgica (sign out). ${ }^{(1,4-7)}$ No sign in, são verificadas a identidade do paciente, a marcação do sítio cirúrgico, a apresentação e assinatura do Termo de Consentimento e a verificação da presença dos materiais solicitados. ${ }^{(8-10)}$ No time out, é feita uma breve pausa antes da incisão, durante a qual todos os membros da equipe se apresentam e expóem sua função na sala, bem como confirmam verbalmente a identificação do paciente, o sítio cirúrgico, o procedimento a ser realizado, a posição do paciente e as possíveis complicações da cirurgia. ${ }^{(8-11)}$ No sing out, os materiais utilizados nas salas operatórias são conferidos e contados, e há o encaminhamento do paciente para a sala de recuperação. ${ }^{(8)}$

Nesse sentido, os benefícios da adesão à Lista de Verificação de Segurança Cirúrgica estão comprovados em estudo de metanálise em que se determinou sua eficácia a partir de três estudos clínicos randomizados controlados (elegíveis e incluídos no estudo), evidenciando redução nas complicações cirúrgicas, como respiratórias (risco relativo de 0,59; intervalo de confiança de 95\% 0,21-1,70; p=0,33), cardíacas (risco relativo de 0,74; intervalo de confiança de $95 \%$ 0,28-1,95; p=0,54) e infecçôes perioperatórias (risco relativo de 0,61 ; intervalo de confiança de 95\% 0,29-1,27; p=0,18), bem como diminuição da mortalidade cirúrgica (risco relativo de 0,59; intervalo de confiança de $95 \% 0,42-0,85) .{ }^{(9)}$

Estudo de revisão sistemática, com 21 estudos de Lista de Verificação de Segurança Cirúrgica implementados nos moldes da OMS e outros dois 
elaborados a partir de experiências institucionais, apontou que o uso da lista era bem compreendido pela equipe cirúrgica, em especial pelos enfermeiros e anestesistas, além de haver divisão de responsabilidades no preenchimento dos itens da lista e boa comunicação e trabalho em equipe. ${ }^{(10)}$ Assim, entre $70 \%$ e $77 \%$ dos profissionais de saúde percebiam que a Lista de Verificação de Segurança Cirúrgica melhorava a comunicação em equipe. ${ }^{(11,12)}$

No que se refere aos custos da implantação das Listas de Verificação de Segurança Cirúrgica, apontado pela revisão sistemática, destaca-se o valor de US $\$ 11,00$ por uso e de US\$ $12.635,00$ pelo treinamento de funcionários com a operacionalização de todos os itens da lista, seja ela nos moldes da OMS ou elaborada pelas próprias instituiçóes de saúde. ${ }^{(10)}$ Esses valores remetem ao ano de 2008. Ainda, nesse estudo, avulta uma percepção da equipe cirúrgica sobre o efeito de atrasos cirúrgicos que a lista causava, uma vez que isso era descortinado como uma ação desnecessária no ato anestésico-cirúrgico. $\mathrm{O}$ preenchimento dos itens de cuidado perioperatório seguro da lista preconizada pela OMS transcorre em cerca de 2 minutos, em média. ${ }^{(13)}$

Embora as evidências demonstrem a significância do uso da Lista de Verificação de Segurança Cirúrgica para a segurança do paciente, estudo de reflexão problematiza que, na operacionalização dessa ferramenta, revela-se uma experiência ainda com falhas e equívocos em seu uso, como a incompletude dos itens de checagem, a baixa adesão, a checagem sem verbalização de seus itens, a fidelidade nos dados preenchidos e a resistência pelos profissionais da saúde. ${ }^{(14)}$ Nesse sentido, os autores ponderam sobre os fatores causais dessa dificuldade, pontuando que a implementação da lista, em alguns serviços de saúde, de forma sumária, passiva e burocrática, representando mais um impresso a ser preenchido, imposto à equipe de enfermagem, possa ter contribuído para a baixa adesão ao seu uso. ${ }^{(14)}$

Considerando a necessidade de se verificar a adesão ao uso da Lista de Verificação de Segurança Cirúrgica, os processos de auditoria vêm ao encontro dessa demanda, pois, nos serviços de saúde, faz-se necessário gerir a qualidade por meio de auditorias de conformidades e educação em saúde, além de conhecer o processo de trabalho multiprofissional, de forma a garantir a mudança comportamental na segurança e na qualidade da assistência cirúrgica. Para que o ambiente cirúrgico seja seguro e confiável, a verificação de critérios para assegurar o cuidado livre de danos, imperícias e eventos que nunca devem ocorrer (never events) se faz necessária.

Assim, optou-se em realizar os critérios de auditoria do Instituto Joanna Briggs (JBI) a partir do curso Evidence-Based Clinical Fellowship Program (EBCFP), realizado pela docente do curso de enfermagem de uma universidade do estado de Santa Catarina, em março de 2018, oferecido pela Escola de Enfermagem da Universidade de São Paulo, o qual preparou profissionais de saúde para promoção do cuidado à saúde baseado em evidências, bem como desenvolver conhecimentos e habilidades de gerenciamento de mudanças e liderança clínica, segundo a metodologia de implementação da colaboração do Centro de Excelência Joanna Briggs. Há estudos que realizaram auditorias independentes e/ou institucionais a partir de departamentos de qualidade e gerenciamento de riscos sobre adesão à Lista de Verificação de Segurança Cirúrgica, porém não se evidenciaram publicaçóes sobre auditorias a partir de critérios auditáveis do JBI, acerca da temática em questão. ${ }^{(15,16)}$ Então, a presente pesquisa buscou responder a seguinte questão norteadora: Há adesão ao uso da Lista de Verificação de Segurança Cirúrgica, pela equipe cirúrgica, a partir dos critérios auditáveis do JBI, para as melhores práticas?

A recomendação para as melhores práticas relativas à efetividade e à adesão da lista de verificação cirúrgica do JBI foi reconhecer que a adesão à lista de verificação cirúrgica depende de sua implementação, pela equipe cirúrgica (Grau de Evidência A) e da educação e do treinamento contínuo sobre os objetivos e seu correto uso, para melhorar sua adesão. Essa educação deve abordar as possíveis percepçôes incorretas em seu uso e a segurança em geral (Grau de Evidência A) e integrar a lista de verificação cirúrgica às rotinas de trabalho existentes, de forma que ela não comprometa e/ou dificulte o processo de trabalho presente/atual (Grau de Evidência A)..$^{(17,18)}$

O objetivo do estudo foi avaliar a adesão ao uso da Lista de Verificação de Segurança Cirúrgica de um hospital referência em cirurgias cardíacas, conforme os critérios auditáveis do JBI. 


\section{Métodos}

Trata-se de estudo observacional, transversal e descritivo. A coleta de dados foi realizada no período de dezembro de 2018 a fevereiro de 2019, com total de 200 (100\%) observaçóes diretas de procedimentos cirúrgicos (gerais, ortopédicos e cardíacos). O cenário de estudo foi o centro cirúrgico de um hospital do oeste do Estado de Santa Catarina, referenciado pelo Ministério da Saúde como atendimento de alta complexidade em cardiologia. $\mathrm{O}$ hospital possuía 174 leitos, com média de 920 internações/mês e de 499 cirurgias/mês. O centro cirúrgico era composto por quatro salas operatórias, sendo uma para as cirurgias cardíacas, a outra para emergências e cesáreas e as demais para outras especialidades médicas.

A Lista de Verificação de Segurança Cirúrgica foi implantada no hospital do estudo em 2013, sendo instituída definitivamente após o treinamento da equipe cirúrgica e inserida no prontuário eletrônico do paciente, nos três momentos operatórios, conforme recomendação da OMS de 2009. Após a implantação, as incongruências contidas na lista eram gerenciadas e discutidas pela coordenadora geral de enfermagem de forma individual com a equipe cirúrgica. Essas inconsistências, às vezes, eram sobre dados de identificaçáo do paciente e itens dos momentos operatórios no sign in, no time out e no sign out. Ainda, o presente local do estudo náo possuía processo de certificação, cujos padróes e requisitos fossem previamente definidos, nacional ou internacionalmente, com vistas à qualidade e à segurança da assistência do paciente atendido, nem tampouco processos auditáveis sobre qualidade de assistência.

Este estudo foi fundamentado na Prática Baseada em Evidência, a qual associa a melhor evidência científica com a experiência clínica, buscando a tomada de decisão crítica e reflexiva para aplicar na assistência à saúde e, assim, implementar, nos serviços de saúde, as melhores práticas. ${ }^{(19)}$ Dentre os centros internacionais que utilizam a Prática Baseada em Evidência, o JBI promove e apoia a síntese, a transferência e a utilização de evidências por meio da identificação de práticas de saúde viáveis, apropriadas, significativas e eficazes para auxiliar na melhoria dos resultados de saúde de forma global. ${ }^{(20)}$

A pesquisa seguiu o método recomendado pelas melhores práticas para adesão ao uso da Lista de
Verificação de Segurança Cirúrgica, sustentado em evidências científicas do JBI, primando pela qualidade dos estudos utilizados, de acordo com seu Grau de Recomendação e seus Níveis de Evidência, sendo estruturado em três fases.

\section{Fase 1 - Auditoria de base}

Previamente ao início da fase de observação, os profissionais foram convidados a participar da pesquisa e assinaram Termo de Consentimento Livre e Esclarecido. Os profissionais eram cirurgióes, anestesistas, enfermeira coordenadora do centro cirúrgico, gerente de enfermagem do hospital e técnicos de enfermagem (circulante e instrumentador cirúrgico). A equipe de auditoria foi estabelecida por esses atores e coordenada por uma acadêmica de enfermagem do Curso de Enfermagem do Centro de Educação Superior da Universidade do Estado de Santa Catarina. Ela foi responsável por medir as conformidades na adesão ao uso à lista de verificação cirúrgica pela equipe multidisciplinar, de acordo com as recomendaçóes das melhores práticas disponibilizadas pelo JBI, com auxílio da docente. A auditoria de base foi realizada pela pesquisadora por 15 dias, após a comunicação à equipe cirúrgica sobre os objetivos do projeto de implementação de melhores práticas. Os dados foram coletados por meio de observaçáo sistemática e não participante, em uma amostra não probabilística, totalizada em cem procedimentos cirúrgicos (geral, ortopédicos e cardíacos). Foram excluídas cirurgias de emergência, devido à preocupação da pesquisadora em não afetar a dinâmica de trabalho da equipe em uma situaçáo de alto risco. Utilizou-se um questionário semiestruturado construído pela pesquisadora a partir do programa JBI Practical Application of Clinical Evidence System (PACES) quanto aos critérios auditáveis em conformidade com a Prática Baseada em Evidência. Os critérios utilizados são apresentados no quadro 1.

\section{Quadro 1. Critérios de auditoria.}

\begin{tabular}{|l|}
\hline $\begin{array}{l}\text { 1. Fortemente recomendado que a lista de verificação envolva toda a equipe cirúrgica. Seu } \\
\text { uso deve ser feito para a melhoria da segurança do paciente }\end{array}$ \\
\hline $\begin{array}{l}\text { 2. Recomendado que a equipe cirúrgica se apresente (fazendo uma pausa) ao paciente } \\
\text { antes da indução anestésica }\end{array}$ \\
\hline $\begin{array}{l}\text { 3. A equipe cirúrgica recebeu educação e treinamento sobre o uso da lista de verificação } \\
\text { cirúrgica. Seu uso é importante na cultura de segurança }\end{array}$ \\
\hline $\begin{array}{l}\text { 4. As listas de verificação cirúrgica são utilizadas para fomentar a cultura de segurança e } \\
\text { não para substituir outras iniciativas que contribuem para essa cultura }\end{array}$ \\
\hline
\end{tabular}

Fonte: traduzido e adaptado de Dias M. JBI Evidence Summary. Evidence-informed practice at the point of care. Surgical safety checklists. The Joanna Briggs Institute EBP Database; 2018. ${ }^{(21)}$ 
A análise de dados descritivos foi realizada por meio de valores percentuais dos critérios auditados conformes e náo conformes, utilizando os programas Microsoft Excel 2013 e Microsoft Word 2013.

\section{Fase 2 - Educativa}

Momento caracterizado pela reflexão sobre os resultados da auditoria de base, elaborando e implementando estratégias para melhorar a prática clínica. Os resultados da auditoria de base foram apresentados à equipe do centro cirúrgico (médicos, anestesistas, enfermeiro e médico coordenador do centro cirúrgico, circulantes e instrumentadores), sendo discutidos os obstáculos e as estratégias propostas para melhorar a conformidade para cada critério auditado. As partes interessadas, incluindo a equipe cirúrgica e a gerente de enfermagem, foram convidadas a participar de rodas de conversa ou conversas individuais ou reunióes na sala de descanso dos colaboradores do centro cirúrgico, para discutir os dados da auditoria de base. Os dados foram apresentados, em gráficos, à equipe cirúrgica e à gerente de enfermagem e, a seguir, foram discutidas as estratégias de melhoria para se obter um aumento no percentual de todos os critérios auditados. A qualidade dos critérios, pelo JBI, deve possuir um percentual acima de 75\%. A ferramenta Getting Research into Practice (GRiP) do JBI foi utilizada para documentar as barreiras encontradas, as estratégias implementadas e os recursos necessários para ultrapassar essas barreiras, objetivando melhorar a conformidade dos critérios auditados. Membros da equipe cirúrgica que possuíam licença médica ou afastamento do trabalho foram excluídos do estudo. Os critérios de inclusão foram todos aqueles profissionais que, no período da coleta de dados, não estiveram em situação de afastamento ou licença.

\section{Fase 3 - Auditoria de seguimento}

Denominada de auditoria pós-implantação, esta foi realizada logo após 1 mês da auditoria de base, por 15 dias consecutivos. Essa fase objetivou avaliar os resultados das intervençóes implementadas. Nos mesmos moldes da auditoria de base, utilizando os mesmos critérios e o instrumento de coleta de da- dos, foram observados cem procedimentos cirúrgicos (geral, ortopédicos e cardíacas), com a mesma equipe cirúrgica da auditoria de base. Os resultados dos percentuais de conformidade de cada critério auditado foram comparados, antes e após a implementação das melhores práticas, permitindo verificar o impacto deste estudo na prática clínica. Os dados foram computados utilizando o Microsoft Excel $^{\circ} 2013$ e o Microsoft Word 2013.

$\mathrm{O}$ estudo atendeu às exigências do Comitê de Ética em Pesquisa Envolvendo Seres Humanos, de acordo com os termos da Resolução Comissão Nacional de Ética em Pesquisa/ Conselho Nacional de Saúde 466/2012, registrado e aprovado sob processo CAAE 88803418.1.0000.0118.

\section{Resultados}

\section{Fase 1 - Auditoria de base}

Os resultados da auditoria de base identificaram que apenas os critérios 2 e 3 tiveram alta conformidades das melhores práticas ( $81 \%$ a $93 \%)$. Os critérios 1 e 4 apresentaram baixa conformidade, variando de $45 \%$ e $49 \%$ e ficando a baixo do valor esperado, que era $75 \%$ (Figura 1 ).

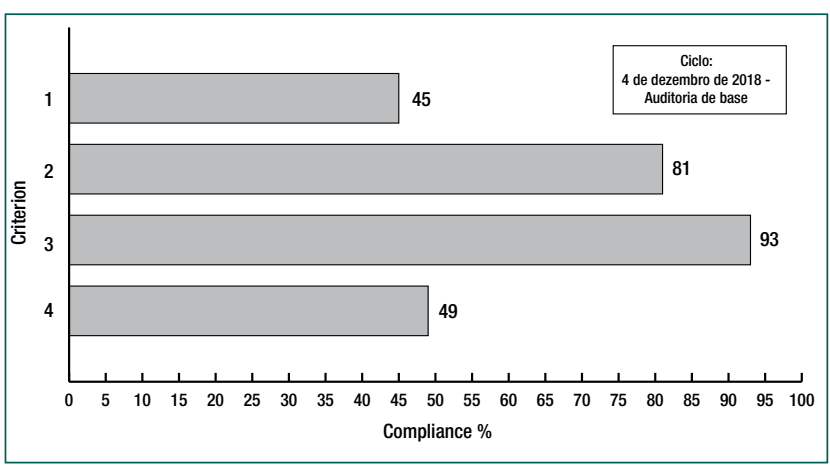

Figura 1. Conformidade com as melhores práticas para a auditoria de base (\%), conforme critérios parametrizados no quadro 1: (1) a implementação da lista de verificação de segurança cirúrgica deve envolver uma equipe multidisciplinar de saúde; (2) a equipe de saúde deve receber educação sobre vários aspectos da lista de verificação e de como usá-la; (3) a lista de verificação de segurança cirúrgica é utilizada para aumentar e não substituir outras iniciativas que contribuam para a cultura de segurança; (4) medidas sensíveis à qualidade da prática da lista de verificação têm sido utilizadas para identificar áreas de melhoria na implementação. 


\section{Fase 2 - Educativa}

Utilizou-se a ferramenta GRiP da JBI para documentar as barreiras encontradas, as estratégias implementadas e os recursos necessários para melhorar a conformidade dos critérios auditados. A fase educativa ocorreu após 7 dias do último dia da auditoria de base, em que a pesquisadora se reuniu, nos períodos da manhá e da tarde, com a gerente de enfermagem, a enfermeira coordenadora do centro cirúrgico, o coordenador médico do centro cirúrgico e coordenador da anestesia. Com a equipe de enfermagem, foi discutida, na sala da gerência de enfermagem do hospital, a inserção continuada da Lista de Verificação de Segurança Cirúrgica no prontuário eletrônico do paciente, pelos funcionários do setor de internaçáo, pois eles eram responsáveis pelo encaminhamento dos pacientes para a recepçáo do centro cirúrgico. Com o coordenador médico do centro cirúrgico, houve um diálogo sobre o incentivo para uma comunicaçáo verbal assertiva e clara do cirurgiáo com o paciente e com a equipe cirúrgica, nos três momentos operatórios. Já com os anestesistas, houve um continuado estímulo das abordagens já realizadas por eles aos pacientes antes da indução anestésica, em que se perguntava sobre alergias, tempo de jejum e o procedimento cirúrgico a ser realizado, sendo feita avaliação da via aérea do paciente. Apesar das náo conformidades resultantes da auditoria de base, os profissionais da cirurgia, da anestesia e da enfermagem mostraram-se proativos e enfáticos para a melhoria da segurança cirúrgica. Todos os membros da equipe foram treinados e reconheceram a importância da comunicaçáo efetiva entre a equipe e a completude do registro da Lista de Verificação de Segurança Cirúrgica.

\section{Fase 3 - Auditoria de seguimento}

Os dados da auditoria de seguimento mostraram-se satisfatórios, pois as taxas de conformidade para todos os critérios auditados tiveram aumento. Especificamente, observaram-se $100 \%$ de conformidade com os critérios relativos à educação e ao treinamento dos profissionais de enfermagem quanto ao uso da Lista de Verificação de Segurança Cirúrgica (Figura 2).

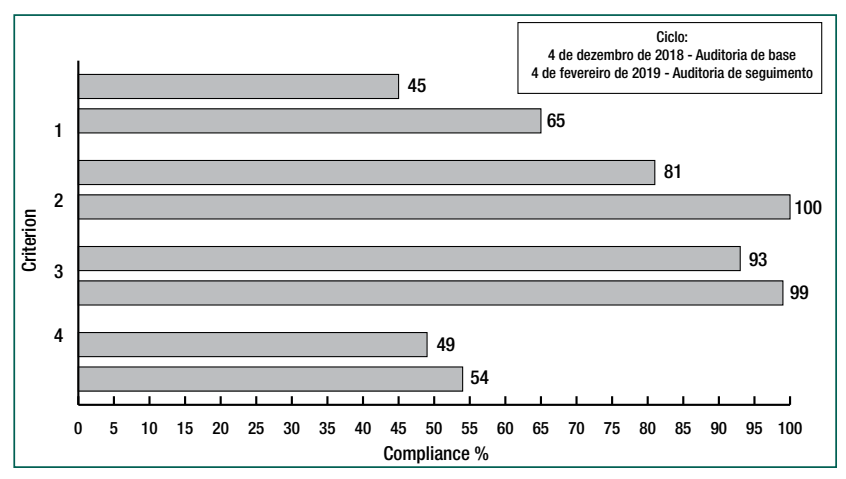

Figura 2. Resultados da auditoria de base e seguimento (\%), conforme critérios parametrizados no quadro 1: (1) a implementação da lista de verificação de segurança cirúrgica deve envolver uma equipe multidisciplinar de saúde; (2) a equipe de saúde deve receber educação sobre vários aspectos da lista de verificação e de como usá-la; (3) a lista de verificação de segurança cirúrgica é utilizada para aumentar e não substituir outras iniciativas que contribuam para a cultura de segurança; (4) medidas sensíveis à qualidade da prática da lista de verificação têm sido utilizadas para identificar áreas de melhoria na implementação.

\section{Discussão}

A auditoria deste estudo avaliou quatro critérios de conformidade das práticas atuais com as melhores evidências disponíveis na literatura. Nossas observaçôes inferem as dificuldades relacionadas à adesão aos procedimentos de cirurgia segura pela equipe multiprofissional (critério 1) e que, possivelmente, influenciam na incompletude do registro eletrônico da Lista de Verificação de Segurança Cirúrgica (critério 4). Diante disso, os achados que estão abaixo do nível de conformidade de $75 \%$ são melhores discutidos para elucidar essa baixa conformidade.

O critério 1 (implantação da Lista de Verificação de Segurança Cirúrgica deve envolver uma equipe de saúde multidisciplinar) revelou baixa conformidade (65\%) na auditoria de seguimento quanto à adesão ao uso da lista. Em 35\% das observaçóes realizadas, não houve identificação da cirurgia segura, nos três tempos operatórios, o que é justificado pelo fato de que esses cirurgióes entraram nas salas operatórias após a indução anestésica e não se comunicaram com a equipe no transoperatório. Considerando a comunicação um aspecto fundamental durante qualquer cirurgia, pondera-se que, entre o tempo da fase educativa e a auditoria de seguimento (15 
dias), eventualmente, não se tenha tido tempo hábil para estruturar os feedbacks junto aos cirurgióes, de modo a se atingir a conformidade desejada.

Estudo realizado na Índia, em que a Lista de Verificação de Segurança Cirúrgica foi auditada em 600 observaçóes (200 no sign in, 200 no sign out e 200 no time out), evidenciou envolvimento ativo dos três membros da equipe cirúrgica (cirurgióes, anestesistas e enfermeiros), em especial no momento operatório do time out $(78 \%)$ da lista. ${ }^{(16)}$ A auditoria desse estudo ocorreu 5 anos após a implantação da lista, pois havia a necessidade de estruturar tanto a equipe como o hospital. Os achados ainda demonstraram que os cirurgiôes utilizaram a lista de verificação, proativamente, nos três momentos cirúrgicos (98,5\% no sign in; $59 \%$ no time out e $88,9 \%$ no sign out). Os itens do time out da lista, nesse estudo, eram sobre a confirmaçáo verbal do paciente e demarcaçáo da lateralidade, a profilaxia antimicrobiana, os exames de imagem e o preenchimento de formulário de patologias. ${ }^{(16)}$ Arguindo ainda sobre a adesão à Lista de Verificação de Segurança Cirúrgica pela equipe cirúrgica, um estudo avaliou, via formulário online, 875 profissionais de saúde (enfermeiros, cirurgiôes e anestesistas) sobre a frequência e a satisfação no uso da lista, após sua implantação em um hospital universitário, constatando-se que 99,4\% dos profissionais avaliados utilizaram a lista. Ainda, $88,3 \%$ especificaram que a manuseiam entre $91 \%$ e $100 \%$ em todas as cirurgias realizadas. ${ }^{(15)} \mathrm{Em}$ uma análise sobre quais profissionais utilizavam mais frequentemente a Lista de Verificaçáo de Segurança Cirúrgica, não houve diferenças significativas entre cirurgiôes e anestesistas $(p=0,212)$ e tampouco entre anestesistas e enfermeiros $(\mathrm{p}=0,451) .{ }^{(15)}$ Assim, esses estudos reforçam a importância do envolvimento multiprofissional, sendo o médico um ator fundamental na mudança de cultura segura nas salas operatórias, uma vez que, sem sua participação ativa, dia a dia, podem ser tomadas decisōes inseguras, até porque a parceria médica é um fenômeno essencialmente interdisciplinar no cuidado cirúrgico. ${ }^{(22)}$

$\mathrm{O}$ envolvimento ativo da equipe cirúrgica, na fase de implementaçáo e na avaliação da Lista de Verificação de Segurança Cirúrgica, é importante, uma vez que se propóem treinamentos contínuos, pressupostos para se obterem a adesão ao uso da lista e a conformidade dos dados. ${ }^{(23,24)}$ Ademais, o treinamento contínuo da equipe do uso da lista pode romper múltiplas barreiras quanto ao uso indevido, ao não uso ou à execução incompleta da lista, e, ainda, o trabalho coletivo parece ser um ponto salutar na eficácia da comunicação.

Nessa perspectiva, os achados do critério 2 da auditoria realizada revelaram que todos os circulantes, em especial os recém-chegados na instituição, receberam treinamento sobre o uso da lista e, ainda, afirmaram que ela é de extrema importância para o andamento cirúrgico, pois náo se pode deixar o paciente sair da sala operatória sem checar todos os itens do sign out, por exemplo. A partir da implantação da Lista de Verificação de Segurança Cirúrgica, as equipes cirúrgicas devem ser capacitadas pelos líderes (hands-on leadership) e, de tempos em tempos, devem-se revisar os protocolos de segurança cirúrgica, para evitar possíveis esquecimentos de seu uso. ${ }^{(25)}$ Faz-se necessário ter um trabalho sistemático de observação e de melhoria do uso da Lista de Verificação de Segurança Cirúrgica, de forma a avaliar seu uso e, caso haja necessidade de alteraçáo de conteúdo, que isso seja realizado para fortalecer a equipe de implementação.

Diante dos aspectos que envolvem a auditoria, salienta-se mencionar que os processos que incorporam o feedback contínuo, em tempo real, são determinantes para que o cuidado seja eficiente, efetivo e seguro. ${ }^{(10,19)}$ Em termos operacionais, estimula-se que o enfermeiro convide os instrumentadores e circulantes, os anestesistas e os cirurgiôes para realizar feedbacks contínuos de conteúdo e execução da Lista de Verificação de Segurança Cirúrgica, além das percepçóes individuais sobre o uso diário da lista. Quando as reunióes presenciais não forem viáveis, o feedback pode ser por e-mail. ${ }^{(26)}$ Assim, vale destacar que a Lista de Verificação de Segurança Cirúrgica é um lembrete útil quando os membros da equipe perioperatória aderem aos protocolos de segurança, porém ela não resolve todos os problemas de segurança - entre eles, a comunicação assertiva e clara entre a equipe nos procedimentos intraoperatórios, a adesáo da equipe aos protocolos de segurança e a redução dos efeitos sentinelas. ${ }^{(27,28)}$ 
Sobre o critério 4 (medidas sensitivas para qualidade de prática da Lista de Verificação de Segurança Cirúrgica têm sido usadas para identificar áreas de melhoria na implementação) da auditoria realizada, evidenciam-se lacunas no preenchimento completo dessa lista, em especial na parte inicial, visto que os setores de internação náo a preencheram. $\mathrm{Na}$ abordagem do nosso processo educativo, houve um diálogo com a gerente de enfermagem sobre essa problemática, apontando possível resolutividade sobre as conferências diárias da lista no prontuário eletrônico, antes de o paciente adentrar a recepção do centro cirúrgico. A taxa de preenchimento da lista, após a auditoria de seguimento, foi de 54\%, estando de acordo com a literatura, que demonstra incompletude dos registros da Lista de Verificaçáo de Segurança Cirúrgica de 76\%, no momento do sign-out, entre as 660 observaçóes cirúrgicas realizadas. ${ }^{(16)}$ Em outro estudo, as taxas de registros completos da Lista de Verificação de Segurança Cirúrgica variaram entre 53,2\% e 60,6\%, com inferência da realização de auditorias de qualidade para possíveis alteraçóes nos itens da lista, bem como um envolvimento constante da equipe cirúrgica, pois percebe-se que cada critério pode favorecer a adesão e, por conseguinte, um ambiente seguro tanto para pacientes como para a equipe cirúrgica. ${ }^{(29)}$

Nessa acepção, é comum haver uma certa incompreensão sobre o uso adequado da Lista de Verificação de Segurança Cirúrgica e quem seria o responsável por perguntar e preencher os itens dela, uma vez que, em geral, os serviços de saúde têm um número significativo de checagens, e a lista pode ocasionar uma fadiga de checagem, reduzindo a confiabilidade dos dados registrados. ${ }^{(23)}$ Soma-se, ainda, uma adoção de treinamentos inadequados ou padronizados por vídeos ou cartazes, que podem ser fatores impeditivos na adesão de seu uso, pois estudos sugerem que treinamentos por equipes, ancorados pela team-based learning, melhoram a percepção, o conhecimento e a conformidade na adesão à execução da lista cirúrgica. ${ }^{(15,30)}$

Ainda que não se tenha proposto discutir o critério 3, por trás da alta conformidade atingida pelos critérios da JBI (93\%), faz-se necessário expressar que a lista fornece à equipe a oportunidade de re- visar eventos e resultados intraoperatório e discutir planos pós-operatórios para uma recuperação bem-sucedida dos pacientes. ${ }^{(31)}$ Para se obterem altos percentuais da conformidade de adesão ao uso da Lista de Verificação de Segurança Cirúrgica, é preciso ter treinamentos contínuos antes, durante e após sua implantação, de forma a oportunizar a qualidade da documentação e a eficiência dos recursos humanos e materiais.

\section{Conclusão}

Os objetivos deste estudo sobre a avaliação da adesão ao uso da Lista de Verificação de Segurança Cirúrgica pela equipe cirúrgica de um hospital de referência cardíaca foram parcialmente alcançados, a partir dos critérios auditados pelo Instituto Joanna Briggs. Ainda, a não obtenção de total conformidade em alguns critérios decorreu do fato de esta ter sido uma auditoria pontual, ou seja, no período de execução do projeto. Náo houve uma pesquisa que avaliasse a adesão ao uso da Lista de Verificação de Segurança Cirúrgica antes de seu processo de implementação. A literatura dá sinais de que, ao longo do tempo, a adesão é possível, desde que existam mais investimentos em treinamentos das equipes cirúrgicas, para se fomentar uma cultura de segurança baseada em evidências e, assim, redefinir esse cenário, com taxas de maior conformidade dos critérios auditáveis. A participação proativa de todos os membros da equipe cirúrgica, em especial do enfermeiro perioperatório, deve ser estabelecida na cultura de segurança, uma vez que nosso estudo não obteve o envolvimento integral do cirurgião no intraoperatório. Por isso, almeja-se o uso de uma abordagem padronizada e sistematizada para nortear a equipe sobre as informaçôes pertinentes do paciente, uma vez que a adesão ao uso de uma lista de verificação potencializa a melhoria na segurança do paciente, à medida que esse indivíduo passa para a próxima fase do tratamento, garantindo que todos os membros da equipe tenham conhecimento dos eventos intraoperatório. Nesse sentido, é possível obter um processo cirúrgico mais seguro, a partir do trabalho em equipe, das estratégias de internalização e de ex- 
pansão, da gestão efetiva de processos e do engajamento integral das pessoas. No geral, o estudo foi avaliado de moderada conformidade, o que fornece elementos fundamentais para sustentar a mudança clínica por meio da prática baseada em evidências. Assim, faz-se necessário realizar adicionais auditorias para manter uma prática clínica pautada nas melhores evidências científicas de cirurgia segura.

\section{Agradecimentos}

O estudo foi financiado pela Universidade do Estado de Santa Catarina por meio do Programa de Apoio ao Ensino de Graduação - PRAPEG (PROEN n.01/2017) em que a pesquisadora realizou o curso Evidence-Based Clinical Fellowship Program (EBCFP) promovido pela Escola de Enfermagem da Universidade de São Paulo (EEUSP). Agradecemos, ainda, a Fundação de Amparo à Pesquisa e Inovação de Santa Catarina (FAPESC), sob o termo de outorga no 2019TR706 - FAPESC 895/2019, que oportunizou o financiamento da divulgação da pesquisa.

\section{Colaborações}

Cabral DB, Pereira MLP, Fernandes MS, Kuczmainski AG e Korb A contribuíram substancialmente com a concepção do estudo, análise e interpretação dos dados, redação e revisão crítica do conteúdo intelectual e aprovação da versão final a ser publicada.

\section{Referências}

1. World Health Organization (WHO). WHO guidelines for safe surgery 2009: safe surgery saves lives [Internet]. Geneva: WHO; 2009. [cited 2020 Jul 29]. Available from: https://www.who.int/patientsafety/ safesurgery/tools_resources/9789241598552/en/

2. Martins GF, Silva AX, Silva Júnior A0, Gomes JA, Gomes TJ. [Patient safety and systematization of nursing care in the postoperative period of cardiac transplantation: a systematic review]. Braz J Health Rev. 2019;2(2):727-41. Portuguese.

3. Carvalho PA, Göttems LB, Pires MR, de Oliveira ML. Safety culture in the operating room of a public hospital in the perception of healthcare professionals. Rev Lat Am Enfermagem. 2015;23(6):1041-8.
4. Panesar SS, Noble DJ, Mirza SB, Patel B, Mann B, Emerton M, et al. Can the surgical checklist reduce the risk of wrong site surgery in orthopaedics? can the checklist help? Supporting evidence from analysis of a national patient incident reporting system. J Orthop Surg Res. 2011;6(1):18.

5. Grigoleto A, Gimenes F, Avelar M. Segurança do cliente e as ações frente ao procedimento cirúrgico. Rev Eletr Enf. 2011;13(2):347-54.

6. Maziero EC, de Camargo Silva AE, de Fátima Mantovani M, de Almeida Cruz ED. [Adherence to the use of the surgical checklist for patient safety]. Rev Gaúcha Enferm. 2015;36(4):14-20. Portuguese.

7. Tostes MF, Galvão CM. Implementation process of the Surgical Safety Checklist: integrative review. Rev Lat Am Enfermagem. 2019;27(3104):e3104.

8. Motta Filho GD, Neves da Silva LF, Ferracini AM, Bähr GL. The WHO Surgical Safety Checklist: knowledge and use by Brazilian orthopedists. Rev Bras Ortop. 2013;48(6):554-62.

9. Biccard BM, Rodseth R, Cronje L, Agaba P, Chikumba E, Du Toit L, et al. A meta-analysis of the efficacy of preoperative surgical safety checklists to improve perioperative outcomes. S Afr Med J. 2016;106(6):592-7.

10. Treadwell JR, Lucas S, Tsou AY. Surgical checklists: a systematic review of impacts and implementation. BMJ Qual Saf. 2014;23(4):299-318.

11. Sewell M, Adebibe M, Jayakumar P, Jowett C, Kong K, Vemulapalli K, et al. Use of the WHO surgical safety checklist in trauma and orthopaedic patients. Int Orthop. 2011;35(6):897-901.

12. Kearns RJ, Uppal V, Bonner J, Robertson J, Daniel M, McGrady EM. The introduction of a surgical safety checklist in a tertiary referral obstetric centre. BMJ Qual Saf. 2011;20(9):818-22.

13. Taylor B, Slater A, Reznick R. The surgical safety checklist effects are sustained, and team culture is strengthened. Surgeon. 2010;8(1):1-4.

14. Tostes MF, Haracemiw A, Mai LD. Surgical Safety Checklist: considerations on institutional policies. Esc Anna Nery. 2016;20(1):2039 .

15. Sendlhofer G, Lumenta DB, Leitgeb K, Kober B, Jantscher L, Schanbacher $M$, et al. The gap between individual perception and compliance: a qualitative follow-up study of the surgical safety checklist application. PLoS One. 2016;11(2):e0149212.

16. Ambulkar R, Ranganathan P, Salunke K, Savarkar S. The World Health Organization Surgical Safety Checklist: an audit of quality of implementation at a tertiary care high volume cancer institution. $J$ Anaesthesiol Clin Pharmacol. 2018;34(3):392-8.

17. Weiser TG, Haynes AB. Ten years of the Surgical Safety Checklist. Br J Surg. 2018;105(8):927-9.

18. Bergs J, Hellings J, Cleemput I, Vandijck D; Flemish Safe Surgery Consortium. The World Health Organisation's surgical safety checklist in Belgian operating theatres: a content-driven evaluation. Acta Chir Belg. 2015;115(2):147-54.

19. Tang R, Ranmuthugala G, Cunningham F. Surgical safety checklists: a review. ANZ J Surg. 2014;84(3):148-54.

20. Camargo FC, Iwamoto HH, Monteiro DA, Lorena LT, Pereira GA. Assessment of an intervention for the diffusion of evidence-based nursing in a teaching hospital. Rev. Gaúcha Enferm. 2016;37(Spe):1-9.

21. Dias M. JBI Evidence Summary. Evidence-informed practice at the point of care. Surgical safety checklists. The Joanna Briggs Institute EBP Database; 2018.

22. Styer KA, Ashley SW, Schmidt I, Zive EM, Eappen S. Implementing the World Health Organization surgical safety checklist: a model for future perioperative initiatives. AORN J. 2011;94(6):590-8. 
23. Levy SM, Senter CE, Hawkins RB, Zhao JY, Doody K, Kao LS, et al. Implementing a surgical checklist: more than checking a box. Surgery. 2012;152(3):331-6

24. Mascherek AC, Schwappach DL, Bezzola P. Frequency of use and knowledge of the WHO-surgical checklist in Swiss hospitals: a crosssectional online survey. Patient Saf Surg. 2013;7(1):36.

25. Püchel VA, Lockwood C. Translating knowledge: Joanna Briggs Institute's expertise. Rev Esc Enferm USP. 2018];52:e03344.

26. Conley DM, Singer SJ, Edmondson L, Berry WR, Gawande AA. Effective surgical safety checklist implementation. J Am Coll Surg. 2011;212(5):873-9.

27. Haugen AS, Murugesh S, Haaverstad R, Eide GE, Søfteland E. A survey of surgical team members' perceptions of near misses and attitudes towards Time Out protocols. BMC Surg. 2013;13(1):46.
28. Ragusa PS, Bitterman A, Auerbach B, Healy WA 3rd. Effectiveness of surgical safety checklists in improving patient safety. Orthopedics. 2016;39(2):e307-10.

29. Mayer EK, Sevdalis N, Rout S, Caris J, Russ S, Mansell J, et al. Surgical checklist implementation project: the impact of variable WHO checklist compliance on risk-adjusted clinical outcomes after national implementation: a longitudinal study. Ann Surg. 2016;263(1):58-63.

30. Rakoff D, Akella K, Guruvegowda C, Chhajwani S, Seshadri S, Sola S. Improved compliance and comprehension of a surgical safety checklist with customized versus standard training: a randomized trial. J Patient Saf. 2018;14(3):138-42.

31. Bartz-Kurycki MA, Anderson KT, Abraham JE, Masada KM, Wang J, Kawaguchi AL, et al. Debriefing: the forgotten phase of the surgical safety checklist. J Surg Res. 2017;213:222-7. 